\title{
Systemic Inflammatory Response Syndrome Associated Alterations in Platelet Indices in Dogs
}

\author{
R. Jasmin ${ }^{1}$, M. Balagangatharathilagar ${ }^{1 * *}$, S. Vairamuthu ${ }^{2}$, D. Sumathi ${ }^{1}$, \\ K. Vijayarani ${ }^{3}$, M.G. Jayathangaraj ${ }^{1}$ and G. Ravikumar ${ }^{4}$ \\ ${ }^{1}$ Department of Veterinary Clinical Medicine, \\ ${ }^{2}$ Centralised Clinical Laboratory, ${ }^{3}$ Department of Animal Biotechnology, \\ Madras Veterinary College, Chennai-600 007, India \\ ${ }^{4}$ Zoonoses Research Laboratory, Madhavaram Milk Colony, Chennai-600 051, \\ Tamil Nadu Veterinary and Animal Sciences University, India \\ **Corresponding author
}

\section{A B S T R A C T}

Systemic Inflammatory Syndrome (SIRS) has been recognized as a life threatening condition in both human and small animal patients admitted in critical care units. Septic

\section{Keywords}

Platelet Indices, Thrombocytopenia, SIRS, DIC, Dogs.

Article Info

Accepted:

26 July 2018

Available Online:

10 August 2018 and non-septic etiologies were documented in the cases with SIRS. Thrombocytopenia and disseminated intravascular coagulopathy (DIC) were consistently observed in many cases with SIRS. Hence, this study was carried out to evaluate the pattern of platelet indices in dogs with Systemic Inflammatory Response Syndrome (SIRS) in comparison with healthy controls. This study evaluated the platelet indices in blood samples obtained from 30 dogs admitted in Critical Care Unit of Madras Veterinary College with SIRS and the results were compared with 10 healthy dogs. Based on physical and hematological examination the dogs with SIRS were classified as SIRS signs with thrombocytopenia $(n=15)$ and SIRS signs without thrombocytopenia $(n=15)$. Blood samples were collected by cephalic venipuncture and analysis was performed using the auto hematology analyzer. The parameters assessed were viz., Mean Platelet Volume (MPV), Platelet Distribution Width (PDW) and Plateletcrit (PCT). The factorial ANOVA model was used for statistical analysis. The results revealed significant variations in platelet indices viz., MPV, PDW and PCT between the healthy dogs and the dogs with SIRS.

\section{Introduction}

Systemic inflammatory response syndrome (SIRS) is a wide spread host response to an infectious as well as non-infectious insults and it is a clinical state rather than a disease entity. If SIRS left untreated, it can lead to multiple organ failure and death (de Laforcade, 2015). Platelet activation during systemic inflammatory conditions contributes to the development of macro- or micro-thrombi, which can result in significant morbidity and mortality in hospitalized patients (Kidd and Mackman, 2013). 
Platelet (PLT) indices are a group of parameters that are used to measure the total amount of PLTs, PLTs morphology and proliferation kinetics (Zhang et al., 2014). Most important parameters among them are plateletcrit (PCT), mean platelet volume (MPV) and platelet distribution width (PDW) (Wiwanitkit, 2004). The average platelet size is described by the MPV and the variability in platelet size by the PDW (Bommer et al., 2008). PCT determines the percentage of the blood volume that consists of platelets (Giacomini et al., 2001).

\section{Materials and Methods}

The study was carried out on the dogs admitted in the Critical Care Unit of Madras Veterinary College Teaching Hospital, Chennai. The dogs were screened for the characteristic signs of SIRS. The dogs presented with two or more of the SIRS criteria (Table 1) as described by Otto (2007) were included in this study.

Hence, the dogs presented with hypothermia, hyperthermia, tachycardia, tachypnea, leukocytosis, leukopenia, thrombocytopenia, progressive multi organ dysfunction syndrome, persistent hypotension were assessed through clinical examination. Blood samples were collected from apparently healthy dogs $(n=10)$ brought for immunization and dogs with SIRS $(n=30)$ by cephalic venipuncture into EDTA anticoagulant tubes and transported to the laboratory immediately.

Mean Platelet Volume (MPV), Platelet Distribution Width (PDW) and Plateletcrit (PCT) analysis were performed using the BC2800 Vet Auto Hematology Analyzer (Mindray Medical Instrumentation).

Selected cases were subjected for further diagnostic tests viz., abdominal ultrasonography, radiography of thorax and abdomen, fine needle aspiration cytology of lumps, examination of peripheral blood smears stained with Giemsa stain for blood parasites, microscopic agglutination test for leptospirosis for diagnosing the existing etiology of SIRS. Based on physical examination and hematological analysis, the dogs were classified into three groups viz.,

Group 1: SIRS signs with thrombocytopenia $(\mathrm{n}=15)$

Group 2: SIRS signs without thrombocytopenia $(n=15)$ and

Group 3: Apparently healthy control $(n=10)$

Statistical analysis was performed using SPSS 12 statistical package.Data were analyzed by using one way ANOVA and the critical difference was estimated by Duncan's test. Receiver's operating curve (ROC) analysis was performed using Medcalc-version 18.5. The sensitivity, specificity and $\mathrm{J}$ index were calculated (Table 4).

\section{Results and Discussion}

Underlying disease identified in thirty SIRS patients are given in table 2 . In the present study number of male animals $(n=19)$ reported were higher than females $(n=11)$.

There was a variety of breeds represented within the study, with the most common being Spitz (8), Non Descripts (5), Labrador Retriever (5), German Shepherd (4), Golden Retriever(3), Doberman (3), Great Dane (1) and Boxer (1). The age represented in this study were ranged from 2 months to 14 years. The results of statistical analysis are given in table 3. In this study we found that platelet count and PCT were decreased whereas MPV and PDW were increased in response to SIRS associated coagulopathy. 
Table.1 Criteria for SIRS in dogs

\begin{tabular}{|c|c|}
\hline Temperature & $<37.2^{\circ} \mathrm{C}$ or $>39.4^{\circ} \mathrm{C}$ \\
\hline Heart Rate (beats/min) & $>150 /$ minute \\
\hline $\begin{array}{l}\text { Respiratory } \\
\text { (breaths/min) }\end{array}$ & $>40 /$ minute \\
\hline WBC count (cells/cmm) & $\begin{array}{l}<5000 \text { or }>19000 \\
>5 \% \text { bands }\end{array}$ \\
\hline
\end{tabular}

Table.2 Etiological diagnosis of SIRS patients

\begin{tabular}{|l|c|l|}
\hline \multicolumn{1}{|c|}{ Clinical Diagnosis } & Number of cases & \multicolumn{1}{c|}{ Diagnostic methods } \\
\hline Ehrlichiosis & 7 & Peripheral blood smear stained with Giemsa stain \\
\hline Skin/Soft tissue infections & 5 & $\begin{array}{l}\text { Deep pyoderma and septic wound during clinical } \\
\text { examination }\end{array}$ \\
\hline Haemorrhagic Enteritis & 4 & $\begin{array}{l}\text { Clinical examination and history of haemorrhagic } \\
\text { diarrhoea }\end{array}$ \\
\hline Snake bite & 4 & $\begin{array}{l}\text { History and clinical examination, presence of fang } \\
\text { marks / edema and necrosis of local tissue }\end{array}$ \\
\hline Pyometra & 3 & Abdominal ultrasound \\
\hline Leptospirosis & 2 & Microscopic Agglutination Test \\
\hline Mammary Tumour & 1 & Fine Needle Aspiration Cytology \\
\hline Prostatic abscess & 1 & Abdominal ultrasound \\
\hline Trypanosomiasis & 1 & $\begin{array}{l}\text { Peripheral blood smear stained with Giemsa stain } \\
\text { and Woo's method }\end{array}$ \\
\hline Ancylostomiasis & 1 & Fecal examination \\
\hline Bebesiosis & 1 & Peripheral blood smear stained with Giemsa stain \\
\hline
\end{tabular}

Table.3 Values of Platelet Indices (Mean \pm SE) in apparently healthy dogs and dogs with SIRS

\begin{tabular}{|c|l|c|c|c|l|}
\hline & Group 1 & \multicolumn{1}{|c|}{ Group 2 } & Group 3 & F value & Significance \\
\hline $\begin{array}{c}\text { PLT count } \\
\text { (lakhs/cmm) }\end{array}$ & $49,133 \pm 7482^{\mathbf{a}}$ & $2,35,000 \pm 21,137^{\mathbf{b}}$ & $3,72,000 \pm 51,358^{\mathbf{c}}$ & $35.38^{* *}$ & $\mathrm{p}<0.000$ \\
\hline MPV( fL) & $8.89 \pm 0.24^{\mathbf{b}}$ & $8.08 \pm 0.23^{\mathbf{a}}$ & $7.66 \pm 0.21^{\mathbf{a}}$ & $6.703^{* *}$ & $\mathrm{p}<0.003$ \\
\hline PDW(\%) & $17.19 \pm 0.20^{\mathbf{c}}$ & $16.48 \pm 0.24^{\mathbf{b}}$ & $15.8 \pm 0.21^{\mathbf{a}}$ & $8.826^{* *}$ & $\mathrm{p}<0.001$ \\
\hline PCT(\%) & $0.05 \pm 0.07^{\mathbf{a}}$ & $0.20 \pm 0.03^{\mathbf{b}}$ & $0.29 \pm 0.04^{\mathbf{c}}$ & $18.74^{* *}$ & $\mathrm{p}<0.000$ \\
\hline
\end{tabular}

${ }^{a, b, c}$ The mean values bearing different superscripts vary significantly 
Table.4 ROC curve analysis (95\% Confidence Interval)

\begin{tabular}{|l|c|c|c|c|c|c|}
\hline & AUC & Z Stat & \multicolumn{1}{c|}{$p$} & Y Index J & $\begin{array}{c}\text { Sensitivity } \\
(\%)\end{array}$ & $\begin{array}{c}\text { Specificity } \\
(\%)\end{array}$ \\
\hline $\begin{array}{l}\text { Platelet Count } \\
\text { (lakhs/cmm) }\end{array}$ & 0.893 & 7.621 & $<0.0001$ & 0.6667 & 76.67 & 90.00 \\
\hline MPV (fl) & 0.747 & 2.931 & 0.003 & 0.4333 & 53.33 & 90.00 \\
\hline PDW (\%) & 0.838 & 5.499 & $<0.0001$ & 0.6667 & 66.67 & 100.00 \\
\hline PCT & 0.870 & 6.590 & $<0.0001$ & 0.6333 & 63.33 & 100.00 \\
\hline
\end{tabular}

Fig.1 Receiver's operating curve analysis for MPV, PDW, PCT and Platelet counts
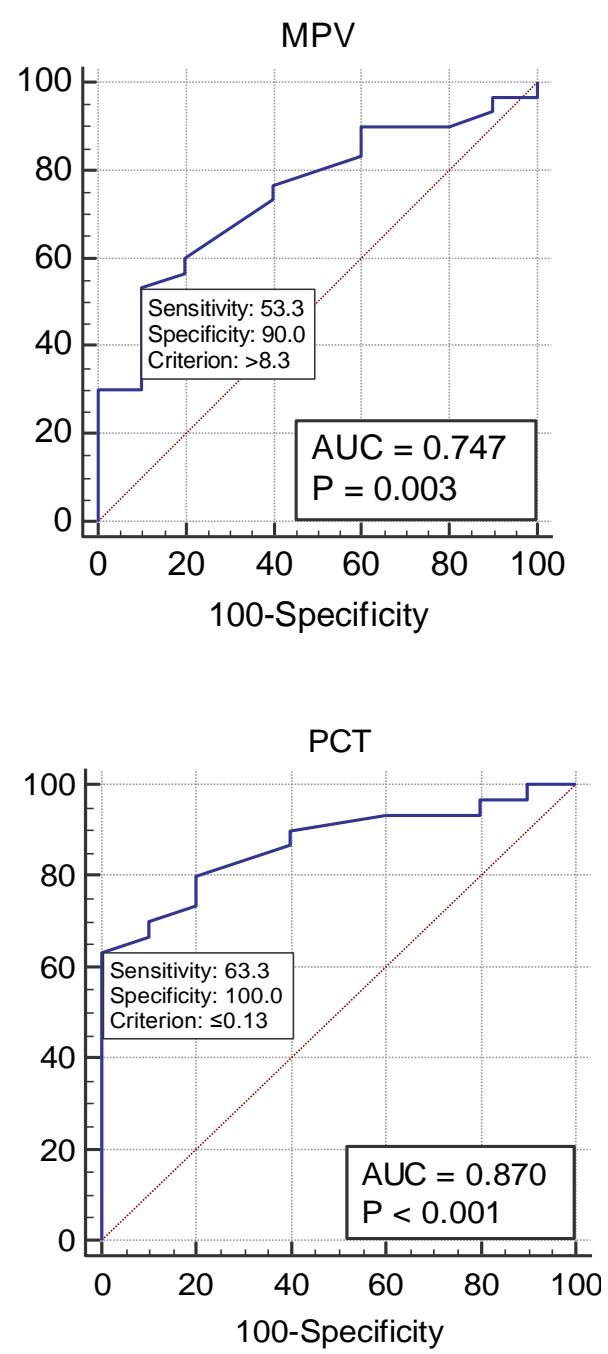

In the present study PLT count, PDW and PCT showed a marked significant difference in between the control and the two groups of SIRS with thrombocytopenia and SIRS without thrombocytopenia whereas MPV

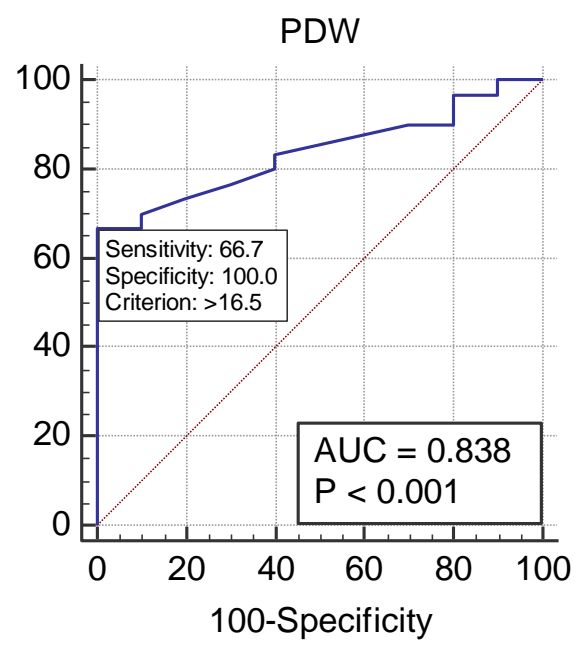

PLT

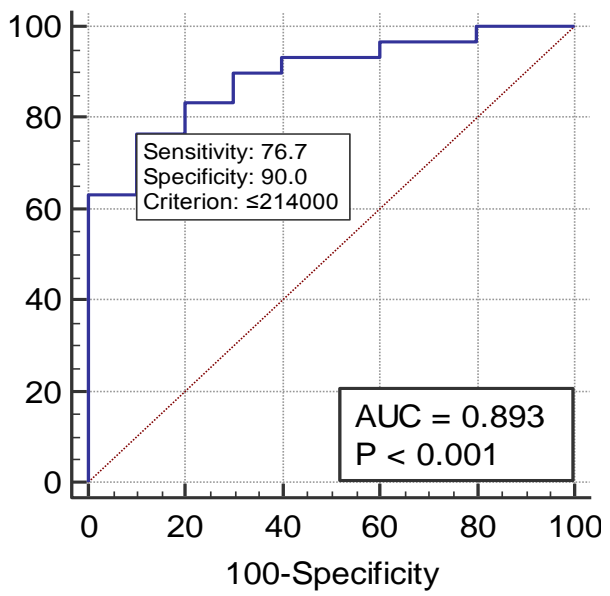

showed marked significance in SIRS with thrombocytopenic group when compared to SIRS without thrombocytopenia and healthy controls. 
Receiver's operating curve analysis (Fig. 1) revealed that the platelet count was found more sensitive $(76.7 \%)$ than MPV, PDW and PCT. Whereas, PDW and PCT were found highly specific $(100 \%)$ in the dogs with SIRS. Thus, the determination of MPV, PDW, PCT in addition to Platelet counts is highly valuable to predict the outcome in canine patients with SIRS.

Inflammatory processes may trigger activation of coagulation by an impaired function of natural anticoagulants (Levi and van der Poll, 2008). Feistritzer and Wiedermann (2007) stated that the activation of hemostasis may finally result in overt disseminated intravascular coagulation (DIC). Reports also indicated that DIC has been associated with increased platelet destruction (Acikgoz et al., 2012). Yilmaz et al., (2008) reported that changes in platelet count and its association with platelet indices may reflect alterations in platelet production and reactivity. Smith et al., (2014) stated that monitoring of platelet activation status in hospitalized patients will be useful for early detection of patients at risk of thromboembolism might allow initiation of thromboprophylaxis and monitoring of the response of platelets to the treatment of underlying disease conditions

The result of the present study suggested that platelet indices are significant parameters in the diagnosis of SIRS associated coagulation disorders. Patients have high risk of death when they are presented with reduced PLT count \& PCT or increased MPV \& PDW as compared to patients with normal platelet indices.

\section{References}

Acikgoz, S., D. Akduman, Z.M. Eskici, M. Can, G. Mungan, B. Guven, F. Comert and V. Sumbuloglu.2012. Thrombocyte and erythrocyte indices in sepsis and disseminated intravascular coagulation. $J \mathrm{Med}$ Biochem. 31:60-64.

Bommer, N.X., D.J. Shaw, E.M. Milne and A.E. Ridyard, 2008. Platelet distribution width and mean platelet volume in the interpretation of thrombocytopenia in dogs. $J$ Small AnimPract49:518-524.

deLaforcade, A., $2015 . \quad$ Systemic Inflammatory Response Syndrome, In Silverstein, D.C and K. Hopper, In textbook of Small Animal Critical Care Medicine, $2^{\text {nd }}$ Edition. Saunders Elsevier, St Louis, pp: 30-34.

Feistritzer, C and C.J. Wiedermann, 2007. Effects of anticoagulant strategies on activation of inflammation and coagulation.ExpetOpinBiolTher. 7:855-870.

Giacomini, A., P. Legovini, G. Gessoni, F. Antico, S. Valverde, M.M. Salvadego and F. Manoni, 2001. Platelet count and parameters determined by the Bayer ADVIATM 120 in reference subjects and patients. Clin Lab Haematol., 23(3): 181-186.

Kidd, L and N. Mackman., 2013. Prothrombotic mechanisms and anticoagulant therapy in dogs with immune-mediated hemolytic anemia. $J$ Vet Emerg Crit Care 2013; 23(1): 313.

Levi, M and T.van der Poll, 2008. The role of natural anticoagulants in the pathogenesis and management of systemic activation of coagulation and inflammation in critically ill patients. Semin Thromb Hemost. 34:459-468.

Otto, 2007. Sepsis in veterinary patients: what do we know and where can we go? $J$ Vet Emerg Crit Care. 17(4):329-332.

Smith, J.R., K.F. Smith and B.M. Brainard, 2014. Platelet parameters from an automated hematology analyzer in dogs with inflammatory clinical 
diseases. Vet J. 201(3):406-411.

Wiwanitkit, V., 2004. Plateletcrit, mean platelet volume, platelet distribution width: its expected values and correlation with parallel red blood cell parameters. ClinAppl Thrombosis/ Hemostasis. 10(2):175-178.

Yilmaz, Z., O. Eralp and Y. Ozarda, 2008. Evaluation of platelet count and its association with plateletcrit, mean platelet volume, and platelet size distribution width in a canine model of endotoxemia. Vet ClinPathol. 37(2): 159-163.

Zhang, Z., Xu, X., Ni, H. and Deng, H., 2014. Platelet indices are novel predictors of hospital mortality in intensive care unit patients. J Crit Care, 29(5): 885.e1-6.

\section{How to cite this article:}

Jasmin, R., M. Balagangatharathilagar, S. Vairamuthu, D. Sumathi, K. Vijayarani, M.G. Jayathangaraj and Ravikumar, G. 2018. Systemic Inflammatory Response Syndrome Associated Alterations in Platelet Indices in Dogs. Int.J.Curr.Microbiol.App.Sci. 7(08): 43844389. doi: https://doi.org/10.20546/ijcmas.2018.708.460 